\title{
Do Ergodic or Chaotic Properties of the Reflection Law Imply Ergodicity or Chaotic Behavior of a Particle's Motion?*
}

\author{
JANUSZ SZCZEPAŃSKI and ELIGIUSZ WAJNRYB
}

Institute of Fundamental Technological Research, Polish Academy of Sciences, Swiętokrzyska 21, 00-049 Warsaw, Poland

(Received 11 June 1994)

\begin{abstract}
The aim of this paper is to answer the question if such properties of reflection law as ergodicity, chaotic behavior and periodicity transfer directly to the motion of a particle in sufficiently large and commonly used classes of the containers. We present two examples. In the first, the above listed properties transfer directly, i.e. ergodicity, periodicity and chaos of the reflection law yield, respectively, ergodicity, periodicity and chaos of the motion but the second example exhibits an opposite relationship: ergodicity and chaotic behavior of the law each imply periodicity of the motion, while periodicity yields ergodicily. These examples show that the answer to the question is negative and the role of the shape of the container is very important even in the case when we assume very strong properties of the reflection laws. Some related macroscopic properties following from the microscopic dynamics are presented, e.g. the properties of the long-time behavior of the distribution function for the corresponding Knudsen gas. Conversely, it turns out that the dynamical systems obtained are closely related to some intensively studied dynamical systems, namely 'standard maps' (topologically conjugated) and one-dimensional (1D) systems. The reflection law corresponding to each standard map is given.
\end{abstract}

\section{INTRODUCTION}

The problem of what happens when the particle reaches the boundary is not only of theoretical interest but of practical importance. This question has been studied for a long time by a number of scientists; up to now there are only hypothesis in this subject, more or less confirmed by experiment.

One of the approaches for studying the behavior of a fluid confined to a container is the approximation that consists of replacing the wall by a smooth surface and assuming that when a particle encounters the wall it 'reflects', that is, its velocity instantaneously changes from its incident value to another 'reflected' value, the latter being such as to take the particle back into the domain of the gas. We call any such transformation of incident velocities into reflected ones a reflection law. Reflection law models were first considered rigorously by Schnute and Shinbrot [1]. They showed that within a reasonable class of reflection laws (in particular, they assumed that a reflection law is a $C^{1}$, isotropic, planar, one-to-one map), there exist only two reflection laws: specular or reverse, such that the fluid does not slip at the boundary. Moreover, under the above assumptions these two reflection laws are the only two for which the entropy function cannot increase for any initial distribution function [2].

Specular reflection is a commonly investigated reflection laws: it states that the angle of incidence is equal to the angle of reflection. If we additionally assume that the gas is very

\footnotetext{
*A part of this paper has been presented at the SIAM Conference on Application of Dynamical Systems, Snowbird, Utah, USA, October 15-19, 1992.
} 
rarefied, i.e. the particles do not interact, then the trajectory of every particle is of billiard type. There are a number of results concerning ergodicity and mixing propertics of classical billiards, i.e. billiards with specular reflection law, and this theory is now being intensively developed. On the other hand, it is well known that some simple mechanical systems are equivalent to billiards in a convex plane domain [3,4]. Moreover, there are interesting physical phenomena like propagation of neutrinos in a medium composed of nuclei [5] or more generally propagation of a test particle through the medium of infinitely many heavy randomly distributed scatterers (mobile [6] or not mobile [7, 8]) which can be understood in some sense as billiards.

In 1984 Babovsky [9] provided a general description of reflection laws in kinetic theory and presented a construction of the solution of the corresponding initial and boundaryvalue problems for a collisionless gas in the case of the Knudsen gas and for interacting particles in the general case of the Boltzmann equation in a bounded region.

The aim of this paper is to investigate the transfer of some interesting (both from a theoretical and practical point of view) properties of the reflection law (boundary conditions) to the dynamics of a particle. To do this it is necessary to assume that in our systems the reflection law is not one-to-one [10]. Then we can investigate ergodic or chaotic reflection laws. The ergodic theorem asserts that the time average of any physical quantity exists for almost all initial conditions and it is equal to the ensemble average. This fact plays a very important role when we make any measurement of a physical quantity for a system in equilibrium. Note that our models are in some sense an intermediate case between the systems first considered by Schnute and Shinbrot and systems with random reflection laws (see [11] and references there).

The following natural questions arise.

$\rightarrow$ Do ergodic (chaotic, periodic) properties of a reflection law imply ergodicity (chaotic, periodic behavior) of the trajectories of the moving particles, respectively?

-What is the influence of the shape of the container?

Some related problems of transferring and the influence of different types of imposed properties of a subsystem into the dynamical system have been studied in [12] (and references therein). It was shown that in the case of horseshoe-like scenario (see also [13]) for a dynamical system the action of contracting, stretching and twist-folding of the phase space leads to a distinct form of dynamics. Implications for ergodicity and turbulence were discussed in connection with Sierpinski and Peano-I Hilbert spaces in 4D. The link between quasi-periodic forcing in case of pendulum and the associated capacity dimension of possible strange attractors were calculated.

Related problems of transfer were also considered in the context of spin systems with long-range interaction [14]. It was shown that under appropriate conditions the inhomogeneous $R$-matrices lead to integrable spin chains.

To give some insight into our problems we consider the motion of a free particle inside two typically used containers: a unit ball in a plane [10] and a square. It turns out that these two systems exhibit quite different properties.

In the first model ergodicity (chaotic behavior) of a reflection law implies ergodicity (chaotic behavior) of the trajectory of a particle and if the reflection law has an attracting periodic orbit then the trajectories of a moving particle are asymptotically periodic. In the second case, ergodicity (or chaotic behavior) of a reflection law leads to periodic properties of the trajectories, while a periodic reflection law leads to ergodic properties of the trajectories.

In both cases we assume that the reflection law is a unimodal map [15] (more specifically a logistic map) and changing the parameter in the reflection law we obtain the required 
qualitative properties. Up to now it is not known what happens when the particle reaches the boundary. The logistic map can be interpreted as a Taylor approximation of the reflection law.

From the other side in our models the logistic reflection law plays a role of coupling for the extended dynamical system describing the particle's motion. Such type of coupling is now extensively studied [16-19] and a number of results (e.g. chaotic behaviour) particularly in the case of coupled map lattices, were obtained. (In [14] several pairwisc potentials are presented which lead to integrable systems.) Notice that most of our results can be generalized to other coupling maps.

One of the physical contexts of the problem investigated in this paper is the single particle dynamics that is recently intensively studied in relation to the motion of a particle in circular accelerators [20]. The Hamiltonian for an electron rotating in a plane perpendicular to a uniform magnetic field is of the form

$$
H=\frac{1}{2} P^{2}-\frac{1}{2 \pi} A \sum_{n=-\infty}^{+\infty} \cos (2 \pi(X-n t)) .
$$

The Poincaré map associated with equation (1) is (see [21])

$$
\begin{aligned}
& X_{n+1}=X_{n}+P_{n+1}, \\
& P_{n+1}=P_{n}+A \sin \left(2 \pi X_{n}\right) .
\end{aligned}
$$

We show that the dynamical systems of this type are equivalent (i.e. topologically conjugated) to the systems considered in our paper for an appropriate reflection law.

It turns out that from the mathematical point of view these two models lead to the following problems.

\section{(a) The first model}

Assume that the map $x_{n+1}=T x_{n}$ exhibits some property (ergodicity, periodicity, etc.) and consider the extended dynamical system

$$
\begin{aligned}
& x_{n+1}=T x_{n}, \\
& y_{n+1}=F\left(x_{n}, y_{n}\right) .
\end{aligned}
$$

Then the question arises under what assumptions on $T$ and $F$ these properties of $T$ transfer to the extended system (3).

The system of this form appears in the paper by Beck [22] and in papers of other authors [23-28] in some physical contexts (Brownian motion) and also as a purely mathematical problem [29]. In [22] a class of nonlinear dynamical systems describing the movement of a particle in a viscous medium under the influence of a kick force is investigated. In this case the time evolution of the velocity of the particle is governed by the system of equations

$$
\begin{aligned}
& x_{n+1}=T x_{n}, \\
& y_{n+1}=\lambda y_{n}+f\left(x_{n}\right) .
\end{aligned}
$$

It was proven [22] that under appropriate conditions on the map $T$, the force $f$ and $\lambda$ $(|\lambda|<1, \lambda$ depends on the viscosity of the liquid) periodicity, ergodicity and the mixing property of $T$ each imply the same property of the extended dynamical system (4). The variable $y$ corresponds here to the velocity of the kicked particle and the ergodic (periodic, mixing) property is supported by the vicinity consisting of other particles (whose evolution is governed by $T$ ) through the force $f$. In our paper ergodicity (periodicity, chaos) is 
transmitted to the velocity of the particle from the boundary of the container by means of the reflection law and then the question is whether each of these properties transfers to the motion of the particle in the whole phase space, i.e. in the space describing the velocity and position of the particle.

In [29] necessary and sufficient conditions for a so called group extension of a minimal (uniquely ergodic) [30] dynamical system to be minimal (uniquely ergodic) are given. It was also shown that in a certain sense a general group extension lifts the above properties.

\section{(b) The second model}

In the following sections we will see that the investigation of the motion of a particle in a square is closely related to the study a map $G$ of the interval $[0, \pi]$, obtained from the reflection law by a suitable translation of the argument; to be more precise, $G=T \circ h$ where $T$ is the reflection law and

$$
h(v)= \begin{cases}v+\pi / 2 & \text { for } 0 \leqslant v<\pi / 2 \\ v-\pi / 2 & \text { for } \pi / 2 \leqslant v \leqslant \pi .\end{cases}
$$

If $T$ is unimodal, $T(0)=T(\pi)$ and $\pi / 2$ is a critical point for $T$ then so is $G$. Similar shaped maps atise in the context of chaotic oscillations in open chemical systems and were studied in [31].

\section{PRELIMINARIES}

To describe the motion of a particle we consider the two-dimensional discrete dynamical system that is obtained from the basic system by considering the position and velocity of the moving particle on the boundary when it hits the wall of a container. (The system obtained in such a way is called the special flow.) In order to get the most simple form of equations of particle motion we use two slightly different systems of co-ordinates in both examples. Therefore, we introduce the following coordinate system: $x_{n}$ denotes the position of the particle on the boundary at the moment of the $n$th reflection, $v_{n}$ is the angle between the velocity of the point before reflection (in the unit ball case, Fig. 1(a)) and after reflection (in the square case, Fig. 1(b)), and the tangent to the boundary at $x_{n}$.

We assume that the law of conservation of energy is valid which implies that we shall restrict our considerations to the case $v \in S^{1}$ ( $S^{1}$ is the unit circle).

\section{THE MOTION IN THE BALL}

In this section we briefly recall the result of [10] for a ball and we present a connection between the so-called 'standard maps' and the system of equations describing the motion of a particle in a ball.

For a ball, these cquations are

$$
\begin{aligned}
& v_{n+1}=T\left(x_{n}, v_{n}\right) \\
& x_{n+1}=x_{n}-2 v_{n+1} \quad(\bmod 2 \pi)
\end{aligned}
$$

where $x_{n} \in[0,2 \pi), v_{n} \in(0, \pi)$.

The second equation is inherently connected with systems of such type and the first constitutes a reflection law. 


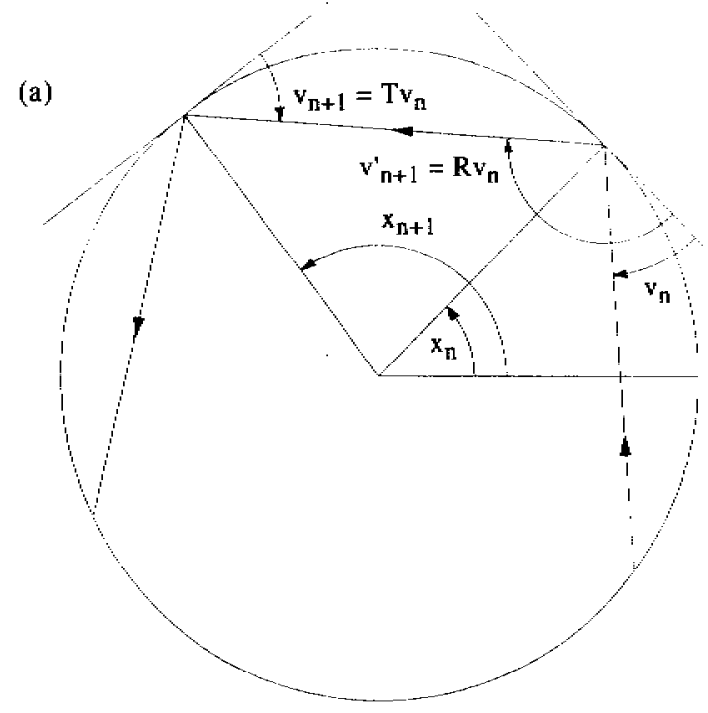

(b)

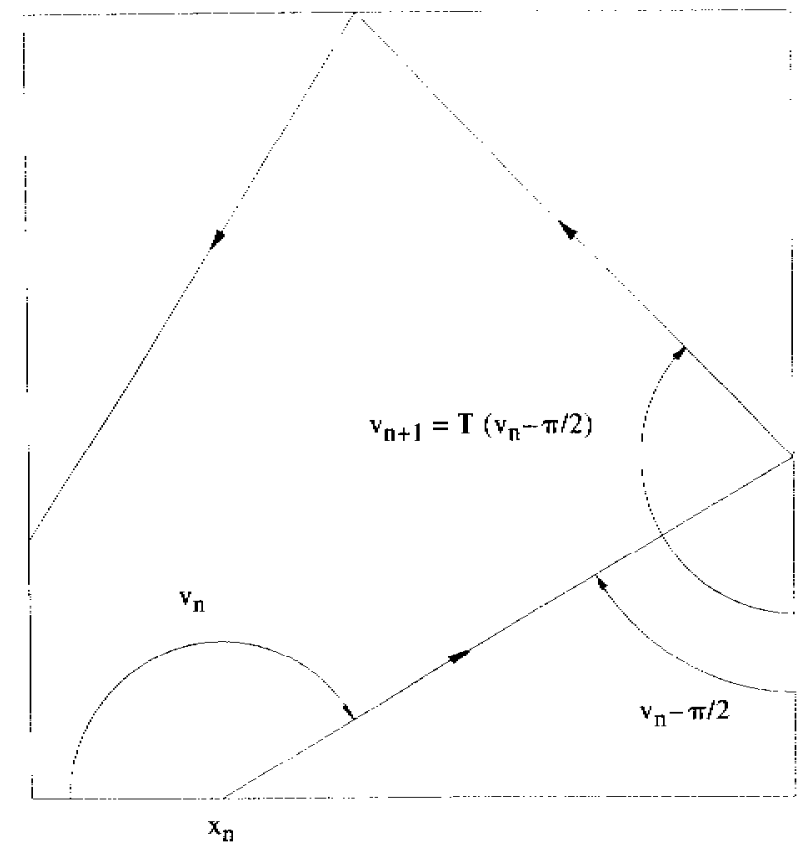

Fig. I. The coordinate system used to describe the motion of a particle: (a) in a ball: here $R$ denotes a reflection law; (b) in a square.

In our considerations we take as reflection law $T$ a logistic map

$$
v_{n+1}=(D / \pi) v_{n}\left(\pi-v_{n}\right) \quad D=4.0 \text {. }
$$

For this parameter $D, T$ is ergodic from $[0, \pi]$ into itself [32]. The study of histograms (Fig. 2) for such a system showed that the extended system is also ergodic.

Now, for the parameter $D$ successively equal to $4.0,3.57,3.59,0.988+\sqrt{8}, 0.995+\sqrt{8}$ 


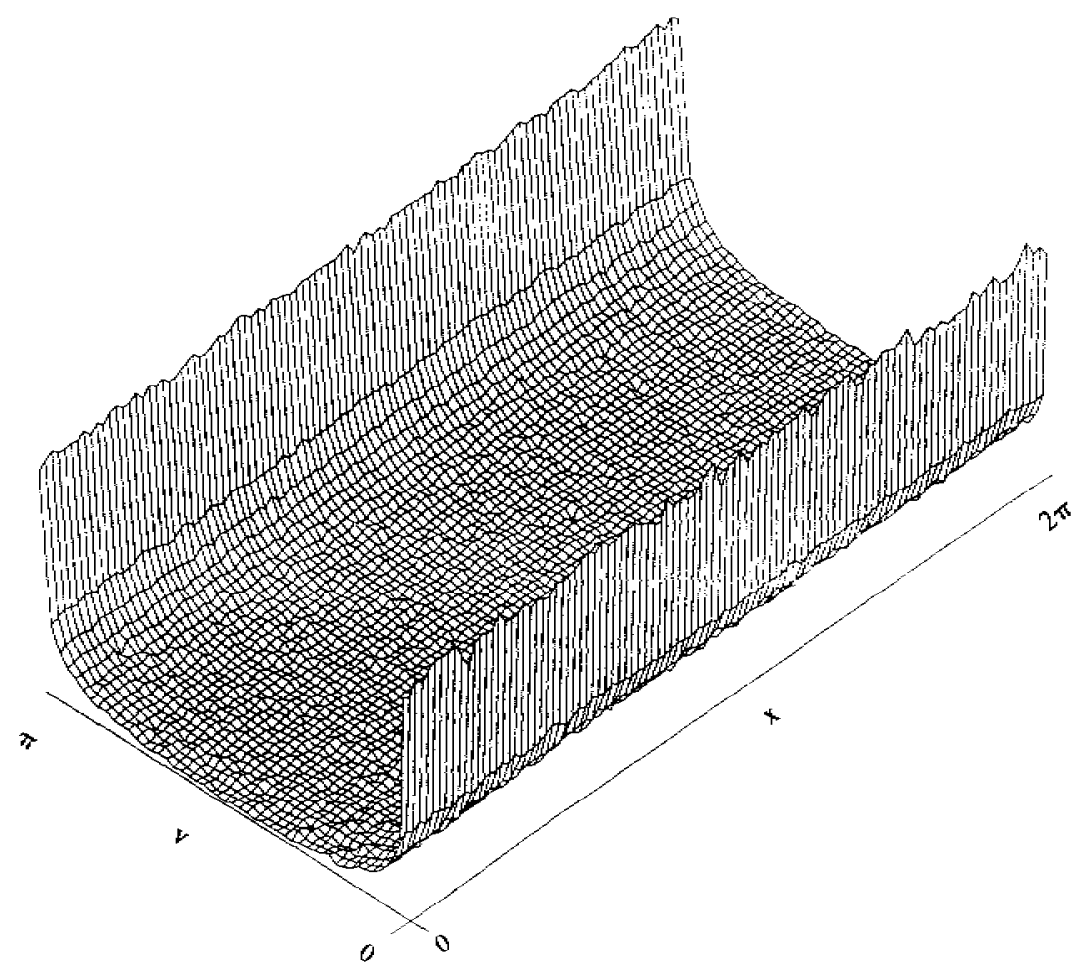

Fig. 2. Histogram for 5000000 iterates of a typical point by the map (6) in 5000 squares (the case of the unit ball) for the reflection law given by (7) and $D=4.0$.

the reflection law $T$ is a chaotic map [32-34] (i.e. the Lyapunov exponent for $T$ is positive [32], hence the trajectories of the dynamical system generated by $T$ exponentially diverge). It follows from the form of the extended dynamical system (6) (with $T$ given by (7)) that one of the Lyapunov exponents of this system has to be equal to the Lyapunov exponent of the base equation $T$, thus the extended system is also chaotic.

Finally, we take $D=2.90007(3.0,3.1)$ so that the reflection law has a stable periodic orbit. Then the trajectories of (6) become regular in the sense that each of them, after a number of reflections, becomes tangent to a finite number of concentric circles and gets asymptotically periodic. These results suggest that ergodicity (chaotic behavior, periodicity) of a reflection law imply the same property of the trajectories. However, the next section shows that this conjecture fails even in the case of a container being a polygon.

From the presented analysis of microscopic behavior of a very rarefied gas, i.e. the Knudsen gas (particles do not interact) it is possible to deduce some properties of the long-time behavior of a one-particle distribution function [10], in particular the loss of analyticity for the Knudsen gas with a periodic reflection law.

It is interesting to observe that the so-called 'standard maps' $[20,21,35-40]$ can be interpreted as dynamical systems describing the motion of a free particle in a ball with an appropriate reflection law. To be more precise, we have checked that the system of the form [40]

$$
\begin{array}{ll}
x_{n+1}=x_{n}+f\left(y_{n}\right) & (\bmod \pi) \\
y_{n+1}=x_{n}+y_{n}+f\left(y_{n}\right) & (\bmod \pi)
\end{array}
$$


( $f$ is a smooth periodic function with period $\pi$ ) is topologically conjugate to the system

$$
\begin{aligned}
v_{n+1} & =v_{n}-\frac{1}{2} f\left(x_{n}\right) & & (\bmod \pi) \\
x_{n+1} & =x_{n}-2 v_{n+1} & & (\bmod 2 \pi)
\end{aligned}
$$

and the conjugation is given by the map

$$
\begin{gathered}
P:[0,2 \pi) \times[0, \pi] \rightarrow[0, \pi) \times[0, \pi] \\
P\left(x_{n}, v_{n}\right):=\left(\pi-\frac{v_{n}}{2}, x_{n}\right)
\end{gathered}
$$

where the first equation in (9) can be interpreted as the reflection law corresponding to the standard map (8).

\section{THE MOTION IN THE SQUARE}

Now we consider the motion of a particle with a non-ideal reflection law in the square. As before we take into account an ergodic reflection law, i.e. we assume $D=4.0$. This reflection law is the same on every wall of the square. In this case our numerical computations show that the trajectories become closer and closer to the walls of the square (Fig. 3) and after some initial collisions with the sides the particles will hit successively the sides of the container counterclockwise.

We now justify this result. First notice that due to the geometry of the square (see Fig. 1(b)) the velocity $v_{n}$ of the particle varies in the following way.

(a) $v_{n+1}=-(4 / \pi) v_{n}\left(\pi-v_{n}\right)$ if particle hits opposite sides; this is only possible when $\pi / 4<v_{n}<(3 / 4) \pi$.

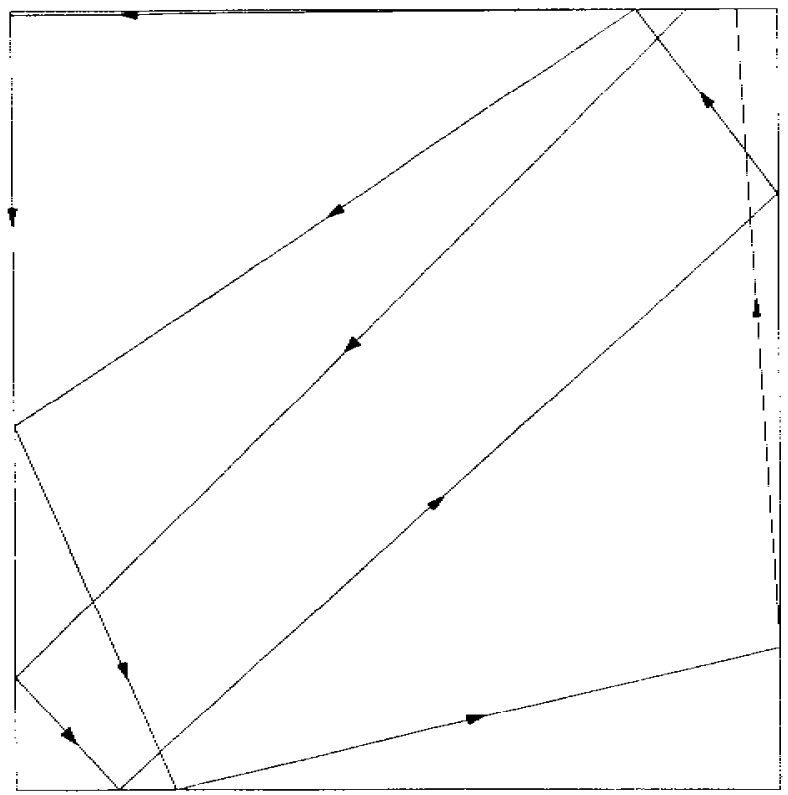

Fig. 3. The trajectory of a typical point for the reflection law with parameter $D=4.0$ (the case of an ergodic and simultaneously chaotic reflection law). 
(b) $v_{n+1}=-(4 / \pi)\left[v_{n}-(\pi / 2)\right]\left[v_{n}+(\pi / 2)\right]$ if the particle hits the sides clockwise; this is only possible when $0<v_{n}<\pi / 2$.

(c) $v_{n+1}=-(4 / \pi)\left[v_{n}-(\pi / 2)\right]\left[v_{n}-(3 / 2) \pi\right]$ if the particle hits the sides counterclockwise; this is only possible when $\pi / 2<v_{n}<\pi$.

Now we notice that if case (a) happens then $\lim _{n} v_{n}=\pi$.

Thus, it is enough to show the following.

Theorem. Consider the map $G:[0, \pi] \rightarrow[0, \pi]$ (Fig. 4) given by

$$
G(v):= \begin{cases}-(4 / \pi)[v-(\pi / 2][v+(\pi / 2)] & \text { for } v \in[0, \pi / 2], \\ -(4 / \pi)[v-(\pi / 2)][v-(3 / 2) \pi] & \text { for } v \in(\pi / 2, \pi] .\end{cases}
$$

Note that $G$ is a unimodal map.

Let $v_{0} \in[0, \pi]$ and $v_{n+1}:=G\left(v_{n}\right)$ for $n=0,1,2,3, \ldots$

$$
\left(v_{n}:=G^{n}\left(v_{0}\right)\right)
$$

Then $\lim _{n} v_{n}=\pi$ for almost all initial velocities $v_{0}$ (with respect to Lebesgue measure $m$ ).

This theorem follows from the more general theorems [15] concerning one-dimensional maps (so called unimodal maps). For completeness we present our proof in the Appendix.

Remark 1. It is easy to notice that the above result holds for maps more general than the unimodal maps.

Remark 2. Using the above method one can show that if $D$ is close to 4.0 then almost all

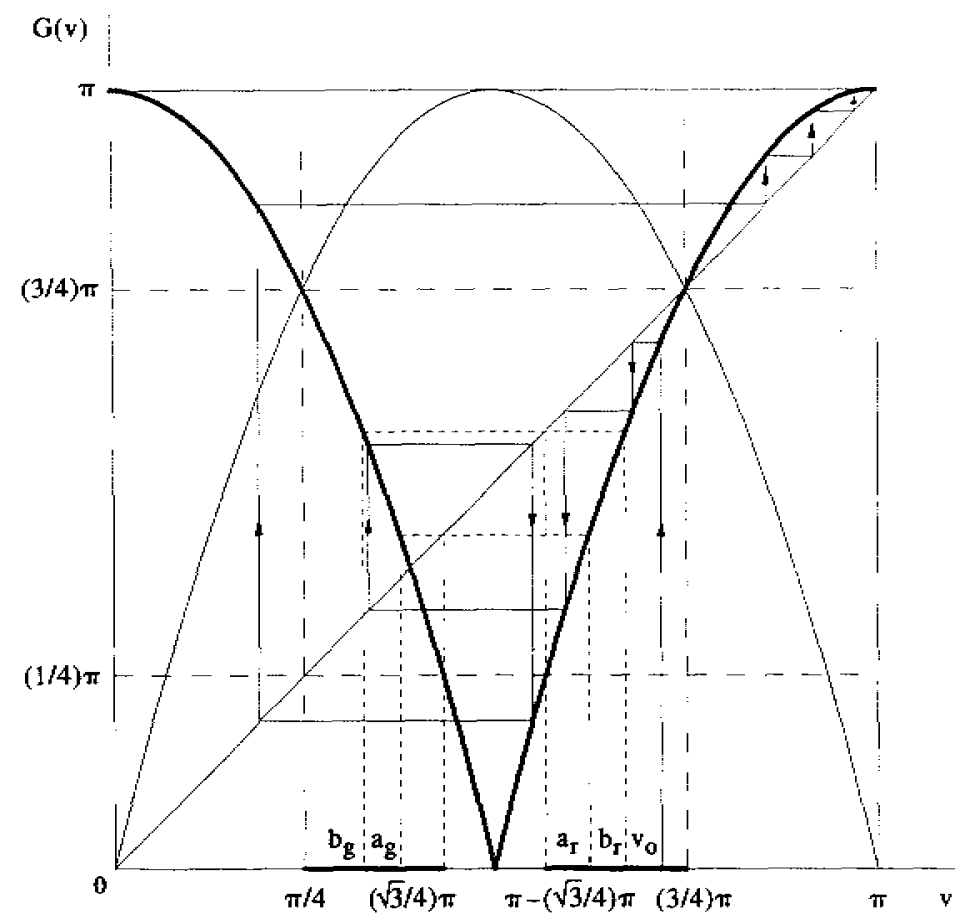

Fig. 4. The graph of $G$. 
points of the interval $[0, \pi]$ tend to a fixed point of the map $G$ and consequently almost all orbits of the particle are also asymptotically periodic.

Remark 3. Note that $G=T \circ h$ where $h$ is an involution, i.e. $h^{2}(v)=v$ for every $v \in$ $[0, \pi]$.

Remark 4. It is well known [32] that for $D=4.0$ the reflection law considered is also chaotic. Hence, even chaotic behavior of a reflection law can lead to an asymptotically periodic motion of the particle.

It follows from the Theorem that for this reflection law an enclave free of particles will appear in the middle of the square (a typical trajectory is presented in Fig. 3). This implies that the distribution function in the position space of the corresponding Knudsen gas loses analyticity independently of the initial distribution function.

Finally, taking $D=2.90007$ (or $3.0,3.1$ ) we get a reflection law with a stable periodic orbit attracting almost all points of the interval $(0, \pi)$. The typical trajectory for this system describing the motion of the particle is shown in Fig. 6. We observe that the trajectory is dense in the configuration space. We also note that the histograms of this dynamical system (see Fig. 5) are independent of the initial point chosen, which is equivalent by the Birkhoff Ergodic Theorem to ergodicity of the system [30]. The graph presented is just the distribution function for invariant measure of the system.

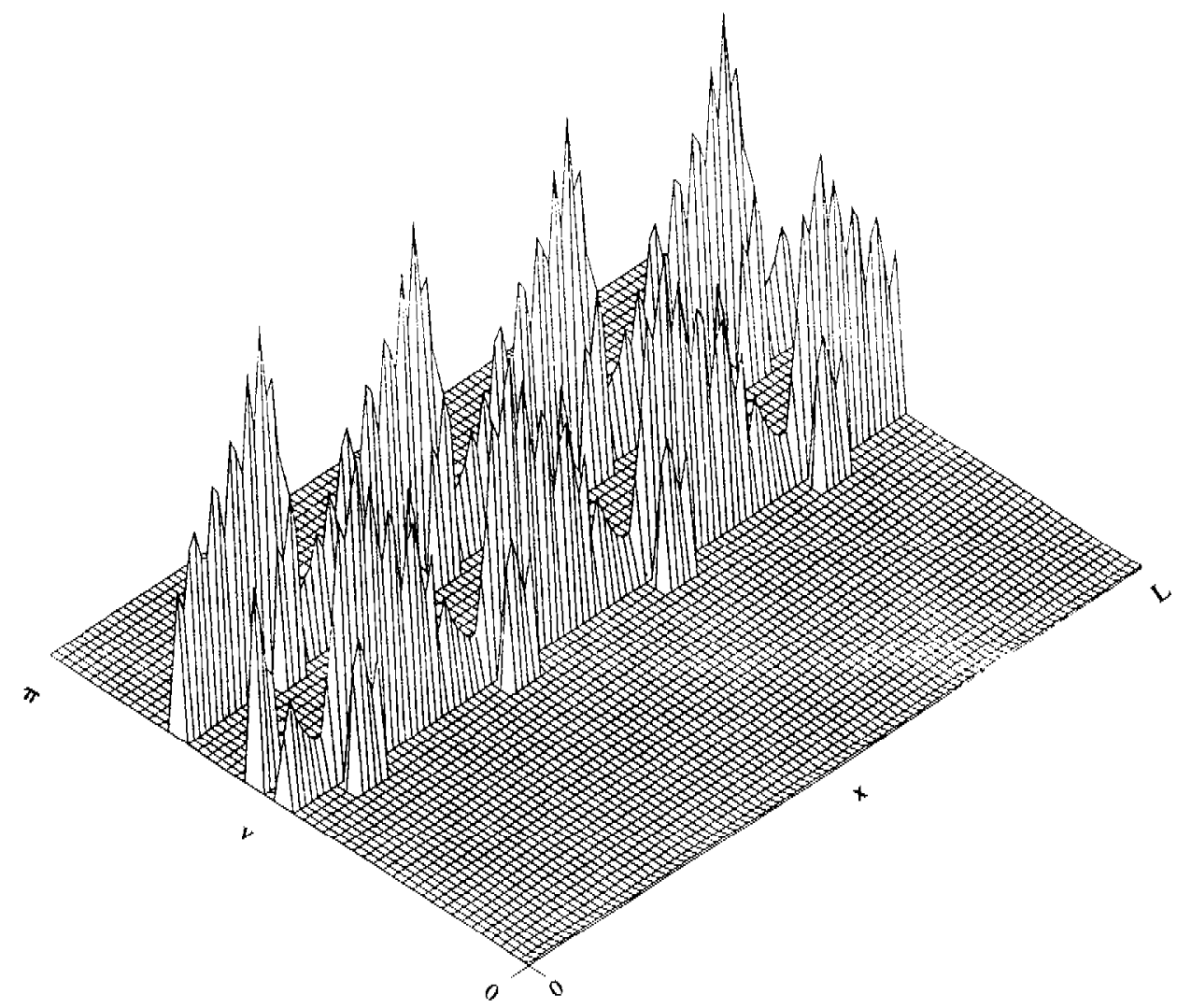

Fig. 5. Histogram for 5000000 iterates of a typical point in a square for reflection law (7) with attracting periodic orbit $(D=2.90007)$. 


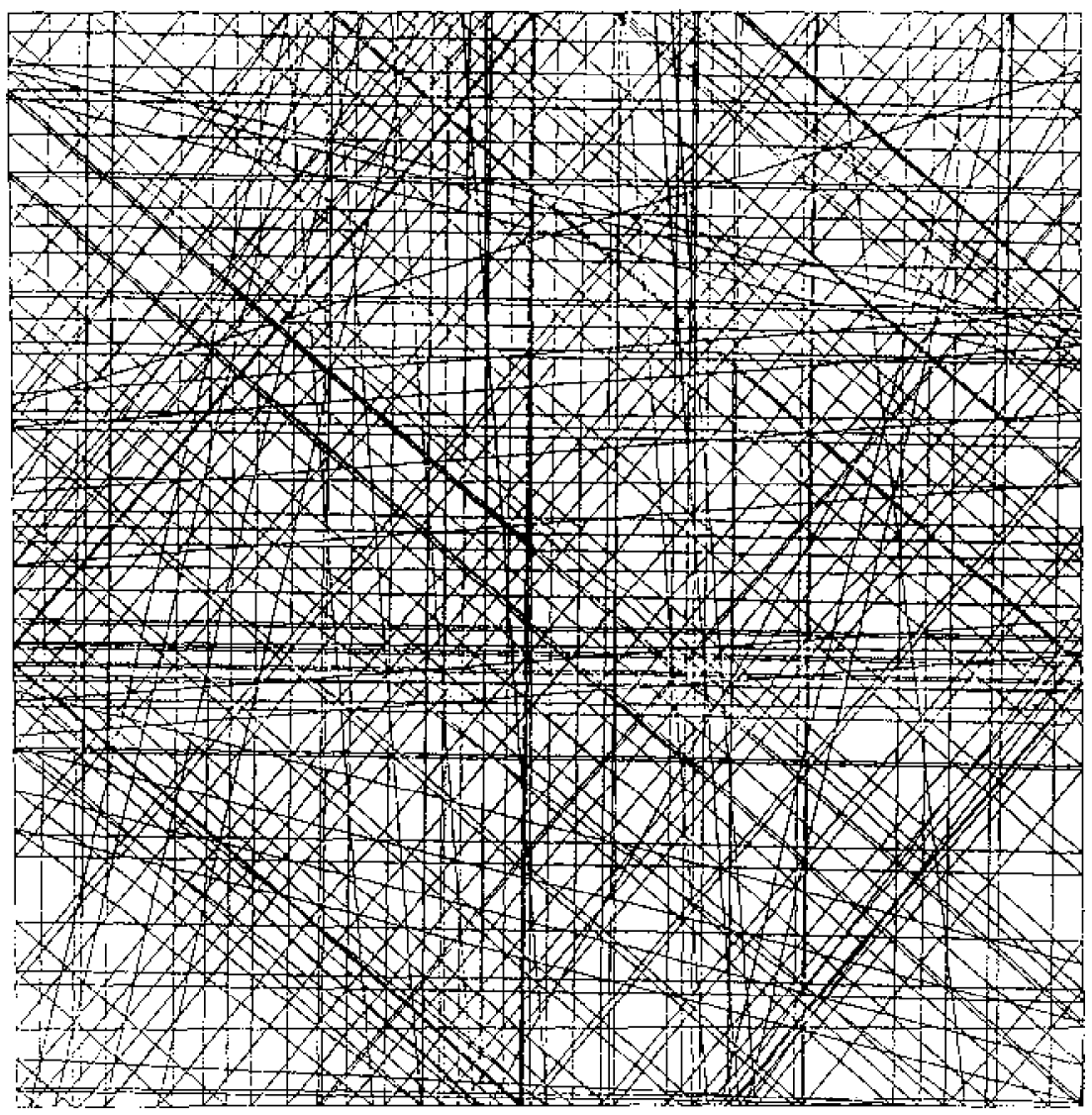

Fig. 6. The trajectory of a typical point in a square for reflection law with attracting periodic orbit $(D=2.90007)$.

\section{FINAL REMARKS}

Our models show that there are no simple relations between properties of a reflection law, the shape of the container and the properties of the motion of the particle. Even very strong properties of the reflection law like ergodicity or chaos do not necessarily transfer to the dynamics of the particle for typically used containers with piecewise of class $C^{\infty}$ boundary (like the square or an arbitrary polygon).

It is an open problem to determine additional assumptions on the reflection law and/or the boundary of the container which would ensure this transfer. It seems that these types of reflection could be interesting from a physical point of view. On the other hand, it turns out that the dynamical systems investigated are in close connection with the systems describing not only the motion of a particle but also other systems like 'standard maps' or even one-dimensional systems.

Acknowledgement-J. S. would like to thank the Kosciuszko Foundation for financial support

\section{KEFERENLES}

1. J. Schnute, M. Shinbrot, Can. J. Math. 25, 1183 (1973).

2. M. Shinbrot, Arch. Rational Mech. Anal. 67, 351 (1978).

3. Ya. G. Sinai, Introduction to Ergodic Theory. Princeton University Press, Princeton (1976).

4. J. Szczepaniski, Bull. Pol. Acad. Tech. 36, 441 (1988). 
5. E. Wajnryb, Acta Phys. Pol. A 65, 107 (1984).

6. J. Piasecki, E. Wajnryb, Physica $A$ 133, 291 (1985).

7. I. Kubo, Nagoya Math. J. 61, 1-57, (1976).

8. L. A. Bunimovich, Ya. G. Sinai, Commun. Math. Phys. 78, 247 (1980); 78, 479 (1981).

9. H. Babovsky, Transp. Theor. Stat. Phys. 13, 455 (1984); 13, 475 (1984).

10. J. Szczepański, E. Wajnryb, Phys. Rev. A 44(6), 3615 (1991).

11. S. Goldstein, C. Kjpnis and N. Ianiro, J. Stat. Phys. 41, 915 (1985).

12. M. S. El Naschie, Il Nuovo Cimento, 107B(5), 583-594 (1992).

13. G. Nicolis, I. Prigogine, Die Erforschung des Komplexen. Piper, Munich (1987).

14. K. Hikami, P. P. Kulish and M. Wadati, Chaos, Solitons \& Fractals, 2(5), 543-550 (1992).

15. P. Collet, J.-P. Eckmann, Iterated Maps on the Interval as Dynamical Systems, edited by A. Jaffe and D. Ruelle. Birkhäuser, Boston (1980).

16. K. Kaneko, Prog. Theor. Phys. 69, 1427 (1983).

17. K. Kaneko, Physica D 23, 436-447 (1986).

18. K. Kaneko, Physica D $\mathbf{3 4}(1,2), 1-41(1989)$.

19. W. Metzler, W. Beau, W. Frees and A. Ueberla, Z. Naturforsch 42A, 310 (1987).

20. J. M. Howett, M. Month and S. Turner, Lecture Notes in Physics, Nonlinear Dynamics, Aspects of Particle Accelerators, Proceedings, Sardinia. Springer, Berlin (1985).

21. Y. H. Ichikawa, T. Kamimura, T. Hatori and S. Y. Kim, Prog. Theor. Phys. Supplement, (98), 1-18 (1989).

22. C. Beck, Commun. Math. Phys. 130, 51-60 (1990).

23. S. Smale, Lecrure Notes in Mathemaits, Vol. 615, p. 48. Springer, Berlin (1977).

24. J. L. Kaplan and J. A. Yorke, Lecture Notes in Mathematics, Vol. 730, p. 204. Springer, Berlin (1979).

25. R. V. Jensen and C. R. Oberman, Phys. Rev. Lett. 46, 1547 (1981)

26. D. Mayer and G. Roepstorff, J. Stat. Phys. 31, 309 (1983).

27. C. Beck and G. Roepstorff, Physica A 145, 1 (1987).

28. T. Shimizu, Phys. Lett. A 140(6), 343-347 (1989).

29. W. Parry, Z. Wahrscheinlichkeitstheorie, verw.Geb., 13, 95-113 (1969).

30. W. Parry, Topics in Ergodic Theory, Cambridge University Piess, Cambridge (1981).

31. M. Misiurewicz and A. L. Kawczyński, Physica D 52, 191-203 (1991).

32. A. J. Lichtenberg and M. A. Lieberman, Regular and Stochastic Motion. Springer, New York (1983).

33. J. E. Hirsch, B. A. Huberman and D. J. Scalpino, Phys. Rev. A 25, 519 (1982).

34. R. Shaw (unpubished).

35. B. V. Chirikov, Phys. Rep. 52, 263 (1979).

36. J. M. Greene, J. Math. Phys. 20, 1183 (1979).

37. D. Bensimon, L. P. Kadanoff, Physica $D$ 13, 82 (1984).

38. R. S. MacKay, J. D. Meiss and I. C. Percival, Physica D 13, 55 (1984).

39. T. Konishi, Prog. Theor. Phys., Supplement, (98), 19 (1989).

40. J. M. Strelcyn, Colloquium Mathematica, 62, FASC.2, 331-345 (1991).

\section{APPENDIX}

\section{Proof of the Theorem}

(The notation used in the Appendix is presented in Fig. 4)

(1) Notice that for any initial velocity

$$
v_{0} \in[0, \pi / 4] \cup[(\sqrt{3} / 4) \pi, \pi-(\sqrt{3} / 4) \pi] \cup[(3 / 4) \pi, \pi], \quad \lim _{n} v_{n}=\pi
$$

(2) Assume that $v_{0} \in[\pi / 4,(\sqrt{3} / 4) \pi] \cup[\pi-(\sqrt{3} / 4) \pi,(3 / 4) \pi]$. We show that for almost every $v_{0}$ there exists $n$ such that $v_{n} \in[(\sqrt{3} / 4) \pi, \pi-(\sqrt{3 / 4}) \pi]$; hence by the above observation we conclude that $\lim _{n} v_{n}=\pi$.

To do this it is enough to show that:

for every

$$
\begin{aligned}
& y_{0}, y_{0}^{\prime} \text { in }[\pi / 4,(\sqrt{3} / 4) \pi] \cup[\pi-(\sqrt{3} / 4) \pi,(3 / 4) \pi] \text { satisfying } \\
& y_{0 n}, y_{0 n}^{\prime} \in[\pi / 4,(\sqrt{3} / 4) \pi] \cup[\pi-(\sqrt{3} / 4) \pi,(3 / 4) \pi] \text { for every } n \in N
\end{aligned}
$$

(where $y_{0 n}:=G^{n}\left(y_{0}\right), y_{0 n}:=G^{n}\left(y_{0}^{\prime}\right)$ ) there exists a subset $A \subset\left[y_{0}, y_{0}^{\prime}\right]$ and a constant $0<c<1$ (independent of $\left.y_{0} . y_{0}^{\prime}\right) \operatorname{such}_{1}$ that $\lim _{n 2} G^{n}(v)=\pi$ for every $v \in A$ and

$$
m(A)>\operatorname{cm}\left(\left[y_{0}, y_{0}\right]\right) \text {. }
$$

(In the case when no such points $y_{0}, y_{0}^{\prime}$ exist the Theorem of course holds).

(a) Assume that for each $n, y_{0 n}$ and $y_{0 m}^{\prime}$ are both in $[\pi / 4,(\sqrt{3} / 4) \pi]$ or both in $[\pi-(\sqrt{3} / 4) \pi,(3 / 4) \pi]$.

By the Lagrange Theorem for every $n \in N$ there is $\xi_{n} \in\left(y_{0}, y_{0}^{\prime}\right)$ with

$$
\left|y_{0 n}-y_{0 n}^{\prime}\right|=\left|G^{n^{\prime}}\left(\xi_{n}\right)\right|\left|y_{0}-y_{0}^{\prime}\right| \text {. }
$$


Since $2<G^{\prime}(\xi)<2 \sqrt{3}$ for $\left.\xi \in[\pi / 4,(\sqrt{3} / 4) \pi] \cup \mid \pi-(\sqrt{3} / 4) \pi,(3 / 4) \pi\right]$, we conclude that the right side of (A3) tends to infinity (because $\left.G^{n^{\prime}}\left(\xi_{n}\right)=\prod_{i=1}^{H=j} G^{\prime}\left(G^{(}\left(\xi_{n}\right)\right)>2^{n}\right)$, which contradicts the fact that the left side is bounded. Thus, for some $n_{i \mathrm{t}}$ the condition ( $\alpha$ ) does not hold. Hence, by (A1) and the Darboux property of $G$,

$$
[(\sqrt{3} / 4) \pi, \pi-(\sqrt{3} / 4) \pi]\left\ulcorner\left[y_{0 m_{4}}, y_{\left(n_{i 1}\right]}\right] \text { for some } n_{(1)}\right. \text {. }
$$

(We chose the smallest one.)

Now we introduce the following notation of the pre-images of the points $(\sqrt{3} / 4) \pi$ and $\pi-(1 / 3 / 4) \pi$ :

$$
G^{-1}((\sqrt{3} / 4) \pi)=\left\{a_{\mathrm{g}}, a_{\mathrm{r}}\right\}, \quad G^{-1}(\pi-(\sqrt{3 / 4}) \pi)=\left\{b_{\mathrm{g}}, b_{\mathrm{r}}\right\}
$$

where $a_{\mathrm{g}}<a_{\mathrm{r}}$ and $b_{\mathrm{g}}<b_{\mathrm{r}}, A_{\mathrm{g}}=\left[b_{\mathrm{g}}, a_{\mathrm{g}}\right], A_{\mathrm{r}}=\left[a_{\mathrm{r}}, b_{\mathrm{r}}\right]$.

We study the pre-images of the interval $A_{\mathrm{T}}$ (the same considerations apply to $A_{\mathrm{p}}$ ).

By (A4) we can assume that

$$
A_{\text {I }} \subseteq\left[G^{\pi_{1}-1}\left(y_{11}\right), G^{n_{\mathrm{R}}-1}\left(y_{0}^{\prime}\right)\right]=\left[y_{\left.0 ! m_{n}-1\right\},}, y_{\left(1, m_{\mathrm{I}}-y_{\mathrm{j}}\right.}^{\prime}\right]
$$

(resp. $A_{g} \subseteq\left[y_{0\left(a_{1}-\mathrm{lj}\right.}, y_{u\left(n_{0}-1\right)}^{\prime}\right]$ ).

Lemma. For each $i=0,1,2, \ldots, n_{0}-1$, the four points $G^{-i}\left(a_{2}\right), G^{-i}\left(b_{c}\right), G^{n_{i}-i-1}\left(y_{0}\right), G^{n_{1}-i \infty} 1\left(y_{0}^{\prime}\right)$ all belong either to $[\pi / 4,(\sqrt{3} / 4) \pi]$ or to $[\pi-(\sqrt{3} / 4) \pi, 3 / 4 \pi]$.

This follows from the fact that the image of the inferval $\left.\left[y_{0}, y_{i}\right]\right]$ under the first $n_{0}-1$ iterations of $G$ does not contain $[(\sqrt{3} / 4) \pi, \pi-(\sqrt{3} / 4) \pi\}$.

Define

$$
\begin{array}{ccrl}
H_{1}:\left[0, \frac{\pi}{2}\right] \rightarrow[0, \pi], & H_{2}:\left[0, \frac{\pi}{2}\right] \rightarrow[0, \pi] \\
H_{1}:=G \mid\left[0 \cdot \frac{\pi}{2}\right]: & H_{2}:=G \mid\left[\frac{\pi}{2}, \pi\right] . \\
G_{1}(v):=\left(-\frac{\pi}{4} v+\frac{\pi^{2}}{4}\right)^{1 / 2}, & G_{1}:[0, \pi] \rightarrow\left[0, \frac{\pi}{2}\right] \\
G_{2}(v):=\pi-\left(-\frac{\pi}{4} v+\frac{\pi^{2}}{4}\right)^{1 / 2} . & G_{2}:[0, \pi] \rightarrow\left[\frac{\pi}{2}, \pi\right] .
\end{array}
$$

$G_{1}$ and $G_{2}$ are left inverses to $H_{1}$ and $H_{2}$ respectively: $G_{1}, H_{1}(v)=v$, for every $v \in[0, \pi / 2]$ and $G_{2}, H_{2}(v)=v$ for every $v \in[\pi / 2, \pi]$.

We shall estintate the ratio

$$
\frac{G^{-\left(\pi_{11}-1\right)}\left(A_{i}\right) \mid}{\left|y_{0}-y_{0}^{\prime}\right|}
$$

where, by definition, $G^{-i}\left(A_{T}\right)=\left\{G^{-i}(\zeta): \zeta \in A_{T}\right\}$ and $G^{-i}(\zeta)=G_{s_{i}}, G_{s_{-1}}, \ldots \circ G_{s_{2}}, G_{s_{1}}(\zeta)$, with $s_{l}$ 이 1 or 2 accordingly as $G^{n_{\mathrm{r}}-i}\left(y_{11}\right) \in[0, \pi / 2]$ or $G^{n_{0}-j}\left(y_{10}\right) \in[\pi / 2, \pi], j=1,2, \ldots, i$. In this definition, we can equally well use $y_{0}^{\prime}$ in place of $y_{n}$ by the Lemma above.

Fitst notice that by the Lagrange Theoren we have

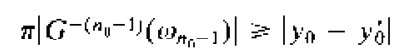

for some $w_{n_{11}} \in G^{n_{11}-1}\left(\left[y_{0}, y_{0}^{t}\right]\right)$.

Hence

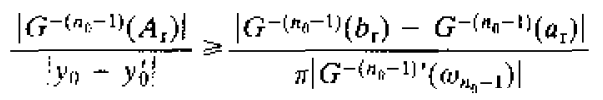

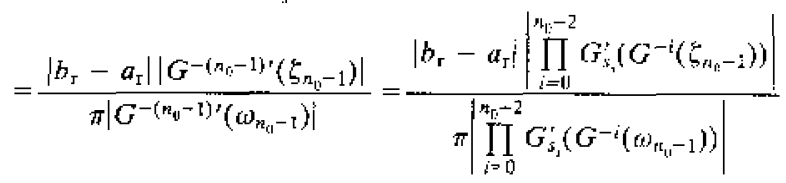

$$
\begin{aligned}
& =\frac{b_{r}-a_{t} \mid}{\pi} \prod_{i=0}^{n_{0}-2} \frac{i G_{s_{i}}^{\prime}\left(G^{-i}\left(\zeta_{n_{0}-1}\right)\right) \mid}{i G_{s_{i}}^{\prime}\left(G^{-i}\left(\omega_{n_{n}-1}\right)\right) \mid}
\end{aligned}
$$

where $E_{n_{i}-1} \omega_{n_{n_{0}-1}} \in(\pi-(\sqrt{3} / 4) \pi,(3 / 4) \pi)$ and for every $i, G^{-i}\left(\zeta_{n_{0}-1}\right), G^{-i}\left(\omega_{n_{n}-1}\right)$ belong to the intervals $G_{s_{i}}([0, \pi])$, where $G_{1}([0, \pi]) \subseteq[\pi / 4,(\sqrt{3} / 4) \pi]$ and $G_{2}([0, \pi]) \subseteq[\pi-(\sqrt{3} / 4) \pi,(3 / 4) \pi]$.

To finish the proof we make use of the following fact.

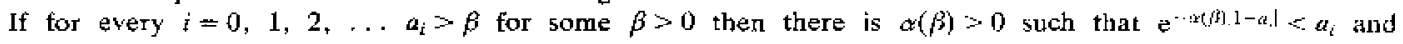


consequently under the notation $p_{n}=\prod_{i=0}^{n} a_{i}, s_{n}=\sum_{i=0}^{n}\left|1-a_{i}\right|$ we have for every $n$, $\mathrm{e}^{-\alpha(\beta) s_{n}<p_{n}}$ and so if $s_{n}<d_{1}<+\infty$ then there is $c_{1}>0$ such that $p_{n}>c_{1}$ for every $n$.

Hence to show that there is $c>0$ such that

$$
\inf _{n_{0}} \prod_{i=0}^{n_{0}-2} \frac{\left|G_{s_{i}}^{\prime}\left(G^{-i}\left(\zeta_{n_{0}-1}\right)\right)\right|}{\left|G_{s_{i}}^{\prime}\left(G^{-i}\left(\omega_{n_{0}-1}\right)\right)\right|} \geqslant c>0
$$

it is enough to prove that there is $d<+\infty$ such that

$$
\sum_{i=0}^{\infty}\left|1-\frac{\left|G_{s_{i}}^{\prime}\left(G^{-i}\left(\zeta_{n_{0}-1}\right)\right)\right|}{\left|G_{s_{i}}^{\prime}\left(G^{-i}\left(\omega_{n_{0}-1}\right)\right)\right|}\right|<d .
$$

Indeed, we have

$$
\begin{aligned}
\sum_{i=0}^{\infty}\left|1-\frac{\left|G_{s_{i}}^{\prime}\left(G^{-i}\left(\zeta_{n_{0}-1}\right)\right)\right|}{\left|G_{s_{i}}^{\prime}\left(G^{-i}\left(\omega_{n_{0}-1}\right)\right)\right|}\right| & <\sum_{i=0}^{\infty} \frac{\left|G_{s_{i}}^{\prime}\left(G^{-i}\left(\omega_{n_{0}-1}\right)\right)-G_{s_{i}}^{\prime}\left(G^{-i}\left(\zeta_{n_{0}-1}\right)\right)\right|}{\left|G_{s_{i}}^{\prime}\left(G^{-i}\left(\omega_{n_{0}-1}\right)\right)\right|} \\
& <\frac{\mathrm{D} \pi}{b_{1}} \sum_{i=0}^{\infty}(\sqrt{3} / 2)^{i}=\frac{D \pi}{b_{1}} \frac{2}{2-\sqrt{3}}<+\infty .
\end{aligned}
$$

Here we have used the fact that for every $\xi, \omega$ that belong either to $[\pi / 4,(\sqrt{3} / 4) \pi]$ or to $[\pi-(\sqrt{3} / 4) \pi,(3 / 4) \pi]$ we have

$$
\left|G_{s}(\xi)-G_{s_{i}}(\omega)\right|<(1 / 2 \sqrt{3})|\xi-\omega|
$$

and consequently

$$
\left|G^{-i}\left(\xi_{n_{0}-1}\right)-G^{-i}\left(\omega_{n_{0}-1}\right)\right|<(1 / 2 \sqrt{ } 3)^{i}\left|\xi_{n_{0}-1}-\omega_{n_{0}-1}\right|,
$$

and the fact that there exists $b_{1}>0$ such that $\left|G_{s_{f}}^{\prime}\left(G^{-i}\left(\omega_{n_{0}-1}\right)\right)\right|>b_{1}$ (since $G^{-i}\left(\omega_{n_{0}-1}\right) \in[\pi / 4,(\sqrt{3} / 4) \pi] \cup$ $[\pi-(\sqrt{ } 3 / 4) \pi,(3 / 4) \pi])$. Furthermore, we have set $D:=\max \left\{\sup G_{1}^{\prime}, \sup G_{2}^{\prime}\right\}<+\infty$.

The estimate obtained is independent of $y_{0}, y_{0}^{\prime}$ thus we have proved the existence of $c>0$ such that (A2) is satisfied.

Removing from the interval $\left[y_{0}, y_{0}^{\prime}\right]$ successfully subintervals satisfying (A2) (i.e. intervals with all points tending to $\pi)$ we conclude that the Lebesgue measure of the union of the removed intervals is equal to $m\left(\left[y_{0}, y_{0}^{\prime}\right]\right)$ (moreover, the set of points that do not tend to $\pi$ has a Cantorlike structure). This implies that for almost every $v_{0} \in[0, \pi], \lim _{n} v_{n}=\pi$, which finishes the proof of the Theorem. 